\title{
Does donor catecholamine administration affect early lung function after transplantation?
}

\author{
M. E. Mukadam, MCh, FRCS (CTh), D. K. Harrington, FRCS, I. C. Wilson, MD, FRCS (CTh), J. G. Mascaro, MD, \\ S. J. Rooney, FRCS (CTh), R. D. Thompson, MRCP, PhD, P. Nightingale, BSc, PhD, and \\ R. S. Bonser, FRCS, FRCP, Birmingham, United Kingdom
}

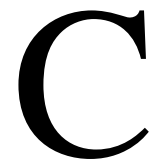

adaveric lung donors have a catecholamine storm of brain death, followed by proinflammatory cytokine response and subsequent pituitary failure. After the storm, a drop in circulating catecholamines and vasoparesis occur, ${ }^{1}$ and exogenous catecholamines are often administered to support systemic vascular resistance and maintain organ perfusion before retrieval.

The exogenous administration of catecholamines (EAC) to organ donors appears to improve outcome after renal and possibly liver transplantation, but in heart transplantation EAC is associated with a worse prognosis. ${ }^{2}$ EAC has been shown to increase alveolar fluid clearance after brainstem death, ${ }^{3}$ but the impact on graft function and recipient outcome after lung transplantation is not known. We hypothesized that EAC might be beneficial in lung transplantation.

\section{Patients and Methods}

We retrospectively analyzed prospectively collected data from 60 consecutive lung transplant donors and recipients (June 1993 to September 2003). The $\mathrm{PaO}_{2} /$ inspired oxygen fraction $\left(\mathrm{FIO}_{2}\right)$ ratios at $\mathrm{FiO}_{2} 1.0$ with $5 \mathrm{~mm}$ positive end-expiratory pressure immediately before retrieval were calculated. Donors were categorized according to catecholamine use from the time of referral for donation to surgery. No EAC was defined as the use of dopamine at less than $2.5 \mu \mathrm{g} /(\mathrm{kg} \cdot \mathrm{min})$ and included those patients receiving vasopressin as a vasoconstrictor. Donors receiving dopamine at 2.5 $\mu \mathrm{g} /(\mathrm{kg} \cdot \mathrm{min})$ or greater, epinephrine, or norepinephrine during the retrieval period were categorized as recipients of EAC. We set a cutoff of less than $2.5 \mu \mathrm{g} /(\mathrm{kg} \cdot \mathrm{min})$ dopamine use because many hemodynamically stable donors receive this dosage per donor hospital protocol.

All recipient operations were conducted with cardiopulmonary bypass. In bilateral lung transplants, reperfusion occurred simultaneously for both lungs. For recipients, similar demographic data

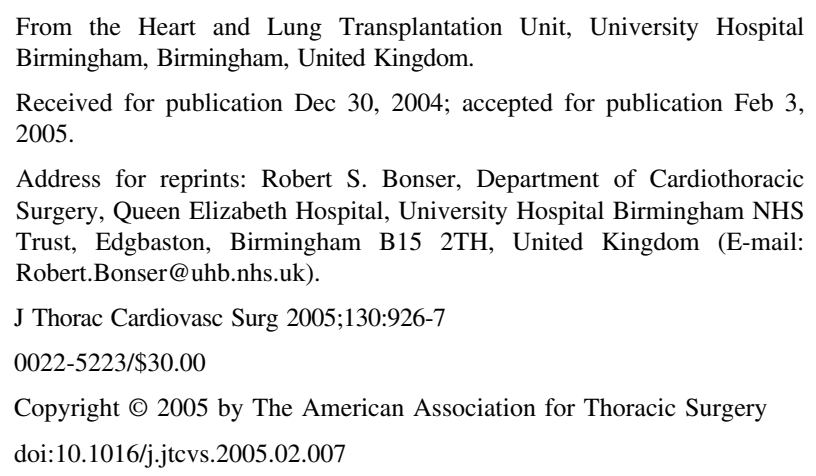

were collected, together with diagnosis, ischemic time, reperfusion strategy, and the $\mathrm{PaO}_{2} / \mathrm{FIO}_{2}$ ratio at 6 hours postimplantation.

Means and SDs were calculated for data with a normal distribution, and medians were calculated for other data. Comparisons were made with paired and unpaired $t$ tests, the Mann-Whitney $U$ test, and the Fisher exact test.

\section{Results}

Sixty donors were evaluated, 29 with EAC and 31 without EAC (Table 1). Donors without EAC were older, but recipients were similarly aged (EAC $52 \pm 10$ years, range 19-63 years, no EAC $54 \pm 7$ years, range 39-63 years, $P=.4$ ). There were no differences in diagnostic category or procedure performed. Traumaticcause donors were more likely to require catecholamine $(P=.03)$. The predominant preservation technique in both groups was pulmonary arterial flush with blood-albumin solution. Controlled reperfusion (limited pulmonary artery pressure of $<20 \mathrm{~mm} \mathrm{Hg}$ for 15 minutes of initial perfusion) and nitric oxide inhalation at implantation (10-20 ppm for the first 6 hours) was nonsignificantly more frequent $(P=.12)$ in the non-EAC group. Ischemic times were similar $(P=.3)$.

Both EAC and non-EAC groups had a fall in $\mathrm{PaO}_{2} / \mathrm{FIO}_{2}$ ratio between retrieval and 6 postoperative hours $(P<.01$; Table 2$)$. This fall was greater in the EAC group $(P=.05)$. The data also suggest a trend toward higher $\mathrm{FIO}_{2}$ and lower $\mathrm{PaO}_{2} / \mathrm{FIO}_{2}$ ratio in this group. The duration of mechanical ventilation was similar (EAC median 3 days, non-EAC median 2 days, $P=.49$ ). On univariate analysis, the change from donor to 6-hour recipient $\mathrm{PaO}_{2} / \mathrm{FIO}_{2}$ did not correlate with ischemic time, preservation technique, operation type, or reperfusion strategy.

\section{Discussion}

This study clearly demonstrates that the process of lung retrieval, transportation, storage, and implantation results in a significant decline in gas exchange after transplantation. Contrary to our initial hypothesis, EAC was associated with worse gas exchange after lung transplantation. This difference was not related to ischemic time, preservation technique, or recipient diagnosis.

This finding is of interest, because we anticipated that EAC might have been protective, as in other noncardiac solid-organ transplantation. Catecholamines may reduce the impact of ischemia-reperfusion injury by modulation of leukocyte adhesion mechanisms ${ }^{4}$ and might be expected to promote alveolar fluid clearance. ${ }^{5}$

A number of possible explanations of our findings in this preliminary study can be proposed. First, it is possible that donors requiring EAC constitute a group with a more severe initial catecholamine storm, potentially resulting in greater direct pulmonary injury or proinflammatory cytokine activation. Alternatively, be- 
TABLE 1. Donor, recipient, preservation, and reperfusion details

\begin{tabular}{|c|c|c|c|}
\hline & $\begin{array}{c}\text { EAC } \\
(n=29)\end{array}$ & $\begin{array}{l}\text { No EAC } \\
(n=31)\end{array}$ & $\begin{array}{c}P \\
\text { value } \\
\end{array}$ \\
\hline Donor age (y, median and range) & $33(11-44)$ & $45(17-59)$ & .002 \\
\hline Cause of brain death (No.) & & & .03 \\
\hline Intracranial hemorrhage & 14 & 25 & \\
\hline Head injury & 10 & 4 & \\
\hline Other & 5 & 2 & \\
\hline Preservation technique (No.) & & & .9 \\
\hline Papworth flush & 20 & 22 & \\
\hline Other flush solution & 6 & 5 & \\
\hline Core cooling & 3 & 4 & \\
\hline Reperfusion strategy (No.) & & & .12 \\
\hline Uncontrolled reperfusion & 21 & 16 & \\
\hline $\begin{array}{l}\text { Controlled reperfusion with } \\
\text { nitric oxide }\end{array}$ & 8 & 15 & \\
\hline Ischemic time (min, mean \pm SD) & $294 \pm 74$ & $273 \pm 69$ & .3 \\
\hline Recipient age (y, mean \pm SD) & $52 \pm 10$ & $54 \pm 7$ & .4 \\
\hline Recipient disease (No.) & & & .9 \\
\hline Emphysema & 18 & 19 & \\
\hline Fibrotic disease & 7 & 6 & \\
\hline Septic disease & 2 & 2 & \\
\hline Other & 2 & 4 & \\
\hline \multicolumn{4}{|l|}{ Type of transplant (No.) } \\
\hline Single lung & 14 & 14 & \\
\hline Bilateral lung & 15 & 17 & $>.999$ \\
\hline $\begin{array}{l}\text { Duration of ventilation ( } d \text {, median } \\
\text { and range) }\end{array}$ & $3(1-140)$ & $2(1-48)$ & .49 \\
\hline
\end{tabular}

cause EAC may have been required to support cardiac function, pulmonary injury may have been exacerbated by elevated left atrial pressure. These findings require confirmation from larger studies.
TABLE 2. Comparisons of gas exchange in donor and recipient

\begin{tabular}{lccc}
\hline & $\begin{array}{c}\text { EAC } \\
(\mathbf{n}=\mathbf{2 9})\end{array}$ & $\begin{array}{c}\text { No EAC } \\
(\mathbf{n}=\mathbf{3 1})\end{array}$ & $\begin{array}{c}\boldsymbol{P} \\
\text { value }\end{array}$ \\
\hline $\begin{array}{l}\text { Donor } \mathrm{PaO}_{2} / \mathrm{FIO}_{2}\left(\mathrm{FiO}_{2} 1.0\right) \\
\text { Recipient } 6 \mathrm{~h} \text { postimplantation }\end{array}$ & $504 \pm 74$ & $486 \pm 86$ & .4 \\
$\quad \mathrm{PaO}_{2}(\mathrm{~mm} \mathrm{Hg})$ & $103 \pm 39$ & $107 \pm 21$ & .59 \\
$\mathrm{FlO}_{2}$ & $0.47 \pm 0.15$ & $0.40 \pm 0.11$ & .07 \\
$\mathrm{PaO}_{2} / \mathrm{FlO}_{2}$ ratio & $244 \pm 111$ & $292 \pm 91$ & .06 \\
$\mathrm{Change}$ from donor $\mathrm{PaO}_{2} / \mathrm{FlO}_{2}$ & $272 \pm 111$ & $200 \pm 137$ & .05
\end{tabular}

All data are mean \pm SD.

\section{Conclusion}

Impairment in early gas exchange is a uniform observation after lung procurement, preservation, and implantation. EAC in the donor was associated with worse posttransplantation gas exchange. Change from donor to 6-hour recipient $\mathrm{PaO}_{2} / \mathrm{FIO}_{2}$ may provide a useful summary assessment of early lung function after implantation.

\section{References}

1. Powner DJ, Darby JM. Current consideration on the issue of brain death. Neurosurgery. 1999;45:1222-6.

2. Schnuelle P, Berger S, de Boer J, Persijn G, van der Woude FJ. Effects of catecholamine application to brain-dead donors on graft survival in solid organ transplantation. Transplantation. 2001;72:455-63.

3. Berthiaume Y, Folkesson HG, Matthay MA. Lung edema clearance: 20 years of progress: invited review: alveolar edema fluid clearance in the injured lung. J Appl Physiol. 2002;93:2207-13.

4. Schnuelle P, Lorenz D, Mueller A, Trede M, Van Der Woude FJ. Donor catecholamine use reduces allograft rejection and improves graft survival after cadaveric renal transplantation. Kidney Int. 1999;56:738-46.

5. Matthay M, Folkesson H, Clerici C. Lung epithelial fluid transport and the resolution of pulmonary edema. Physiol Rev. 2002;82:569-600. 